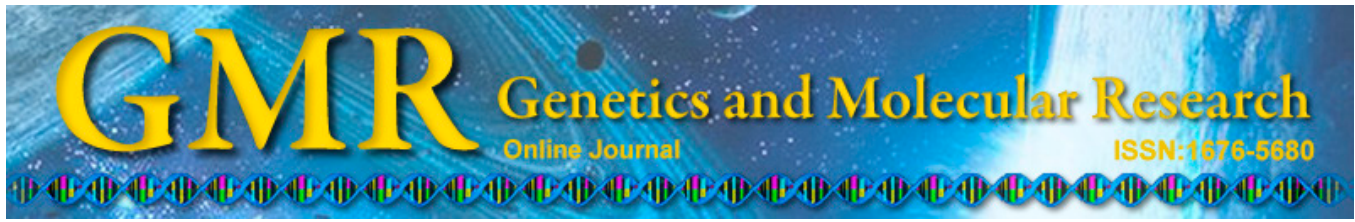

\title{
Screening of rice (Oryza sativa L.) OsPR1b- interacting factors and their roles in resisting bacterial blight
}

\author{
Z.H. Luan ${ }^{1,2}$ and D.W. Zhou ${ }^{3}$ \\ ${ }^{1}$ Institute of Grassland Science, Northeast Normal University, \\ Key Laboratory of Vegetation Ecology, Ministry of Education, \\ Northeast Normal University, Changchun, China \\ ${ }^{2}$ Biology Department, Tonghua Normal University, Tonghua, China \\ ${ }^{3}$ Northeast Institute of Geography and Agroecology, \\ Chinese Academy of Sciences, Jilin, China \\ Corresponding author: D.W. Zhou \\ E-mail: zhoudwniga@163.com
}

Genet. Mol. Res. 14 (1): 1868-1874 (2015)

Received November 11, 2014

Accepted February 19, 2015

Published March 13, 2015

DOI http://dx.doi.org/10.4238/2015.March.13.15

\begin{abstract}
PR genes, a type of genetic marker, are constitutively expressed at background levels, while being easily inducible by pathogenic bacteria. By using a yeast two-hybrid technique, four rice (Oryza sativa L.) OsPR1b-interacting factors were screened. Homozygous plants overexpressing OsPR1b were prepared by transgenic technology. We postulated that OsPR1b may participate in the resistance signaling pathway of rice. Of simultaneous treatments with hormones and pathogenic bacteria, exogenously applying JA and ET significantly increased the expression level of OsPR1b genes in seedlings. Compared with the control group that was inoculated with water, inoculation with a mixture of water and pathogenic bacteria hardly affected the expression level of OsPR1b gene, while cotreatment with SA and pathogenic bacteria slightly upregulated the expression level. However, cotreatment with JA or ET and pathogenic bacteria
\end{abstract}


managed to significantly upregulate the expression level of the OsPR $1 \mathrm{~b}$ gene by 4.8 or 5.7 fold. PR genes, which are sensitive, are prone to many unknown factors during expression, and the detailed regulatory mechanisms in rice still require in-depth studies.

Key words: Rice bacterial blight; Yeast two-hybrid; Hormone treatment

\section{INTRODUCTION}

Rice (Oryza sativa L.) is one the most important cereal crops worldwide, but the single-cropping pattern in which it is grown renders it prone to damage during growth, such as by rice bacterial blight, which is induced by the Gram-negative Xanthomonas oryzae pv. oryzae (Xoo) bacteria (Gnanamanickam et al., 1999). Thus, the study of resistance genes and the development of resistant varieties is currently the most effective method of long-term disease prevention and treatment.

Two PR genes are present in rice: PR1a and PR1b, which code for the PR1a acidic protein and PR1b basic protein, respectively. Their expression is induced by various environmental conditions, as well as by some chemical substances (Agrawal et al., 2001). Exogenously applying JA and ET can induce an intense SAR reaction at roots, accompanied by the significantly upregulated expression of PR1a and PR1b (Nahar et al., 2011). Since the induced expression of PR genes is able to enhance the plant's disease-resistance, the method has been widely used in the study of characteristic molecular markers in rice defense-related studies.

In this study, OsPR1b (Os01g28450), a pathogenesis-related gene, was selected to be screened for interacting factors by employing a yeast two-hybrid technique. Gene expression after hormone treatment was detected, with the aim of identifying interacting factors in the induced resistance signaling pathway. The results provide valuable information for the clarification of the biological functions of OsPR1b, and the complicated regulating effects acting on bacterial blight.

\section{MATERIAL AND METHODS}

\section{Yeast two-hybrid assay}

Specific primers OsPR1b_F and OsPR1b_R (Table 1) were amplified as OsPR1b, then then spliced into PGEM-T (Promega). Correctly cloned pGBK-OsPR1b was obtained by sequencing and screening. According to the instructions of Yeastmaker ${ }^{\mathrm{TM}}$ Yeast Transformation System 2, the recombinant bait plasmid was transformed into the GOLD yeast strain, then streaked onto aSD/-Trp/X- $\alpha$-Gal plate that were then cultured at $30^{\circ} \mathrm{C}$ for $3-5$ days, to observe the growth of yeast colonies. Two yeasts containing bait proteins both with and without selfactivation activity were used as the positive and negative controls, respectively.

\begin{tabular}{|c|c|c|c|}
\hline Primer's name & Sequence $\left(5^{\prime}-3^{\prime}\right)$ & Primer's name & Sequence (5'-3') \\
\hline OsPR1b_F & GTGGCGGAGAAGCAGTGGTA & OsPR1b_R & ACGGCTGACGGCTTTATTCC \\
\hline OsActin_F & GAGTATGATGAGTCGGGTCCAG & OsActin_R & ACACCAACAATCCCAAACAGAG \\
\hline OsPR1b_RT_F & ACGCCTTCACGGTCCATAC & OsPR1b_RT_R & AAACAGAAAGAACAGAGGAGTA \\
\hline
\end{tabular}


Total RNA was extracted from the leaves of rice using the Trizol method, and a cDNA expression library was established in the Y187 yeast strain using the FastTrack 2.0 kit (Invitrogen). Then, $10 \mu \mathrm{L}$ of the $5 \mathrm{~mL}$ yeast cDNA library was collected and diluted 1000 times, 50 $\mu \mathrm{L}$ of which was then used to coat a $\mathrm{SD} /$-Leu plate that was cultured at $30^{\circ} \mathrm{C}$ overnight, after which the number of clones was counted. The library titer was calculated according to "library titer $(\mathrm{cfu} / \mathrm{mL})=$ colony count $\mathrm{x}$ dilution factor", volume of coated plate $(\mathrm{mL})$ and "total capacity of library $(\mathrm{cfu})=$ average library titer $(\mathrm{cfu} / \mathrm{mL})$ x total volume of library $(\mathrm{mL}) "$."

According to the operating manual of Clontech, library screening was performed by matching pairs between the GOLD yeast strain containing pGBK-OsPR1b plasmid, and the Y187 yeast strain containing the rice total cDNA library. The screened single colonies were identified by colorimetric methods, and plasmids were extracted for self-activation detection, in order to exclude false positive results. Positive clones were inserted into sequences for alignment.

\section{Vector construction and plant transformation}

By using the total DNA of Nipponbare rice as the template, OsPR1b was obtained by specific amplification and sliced into the pMD19 vector. Subsequently, the resulting vector was transformed into an Agrobacterium bacterium EHA105 by electronic shock, and then into the callus tissues of Ishikari-Shiroke using Hiei's transgenic method. The $\mathrm{T}_{1}, \mathrm{~T}_{2}$, and $\mathrm{T}_{3}$ generations of $\mathrm{T}_{0}$ generation plants that were detected as positive by PCR were also detected as positive. $\mathrm{T}_{3}$ generation (3:1) homozygous plants overexpressing OsPR1b were saved for further use.

\section{Plant breeding and inoculation of pathogenic bacteria}

Seeds were germinated at $37^{\circ} \mathrm{C}$, and grown hydroponically in a greenhouse [approximately $200 \mu \mathrm{M}$ photons $\mathrm{m}^{-2 \mathrm{~s}-1}$, humidity at $55 \%, 12$-h light $\left(32^{\circ} \mathrm{C}\right) / 12 \mathrm{~h}$ dark $\left.\left(28^{\circ} \mathrm{C}\right)\right]$. To clarify the interaction between rice pathogenic bacteria and the OsPR1b gene, the leaves of two-week-old rice seedlings were cut and inoculated with Xoo P8.

\section{Semi-quantitative PCR and quantitative PCR}

Semi-quantitative PCR was conducted using OsPR1b_RT_F and OsPR1b_RT_R (Table 1) as primers, as well as OsActin (OsActin_F and OsActin_R, Table 1) as the internal reference gene.

The reaction system for quantitative PCR was prepared according to the instructions provided with the SsoFast EvaGreen Supermix kit. Conditions for amplification were as follows: $95^{\circ} \mathrm{C}$ for $5 \mathrm{~min}, 95^{\circ} \mathrm{C}$ for $10 \mathrm{~s}, 58^{\circ} \mathrm{C}$ for $10 \mathrm{~s}, 72^{\circ} \mathrm{C}$ for $20 \mathrm{~s}$ and $72^{\circ} \mathrm{C}$ for $10 \mathrm{~min}$, for 45 cycles in total. LightCycler 480 real-time PCR was used throughout the experiment. Housekeeping gene OsActin was employed as the internal standard at the correct template amount, based on $2^{-\Delta(\Delta \mathrm{Cp})}$. Each experiment was performed in triplicate.

\section{RESULTS}

\section{Screening of host interacting factors}

To analyze whether the expression of a gene was toxic to a yeast strain (i.e., whether it was an eligible candidate bait protein), and to verify the self-activation activity of OsPR1b, the 
GOLD yeast strain that expressed pGBK-OsPR1b was grown in SD/-Trp/X- $\alpha$-Gal culture medium. The GOLD yeast strain containing OsPR1b protein grew similarly to the control group, suggesting that the OsPR1b protein has no toxic effects on the growth of this strain (Figure 1). Unlike the control strain that expressed the self-activation protein, neither the GOLD yeast strain expressing OsPR1b nor the control which did not express the self-activation protein was blue on the SD/-Trp/X- $\alpha-$ Gal plate. Therefore, OsPR $1 b$ alone could not activate the expression of the reporter gene, namely, OsPR1b had no self-activation activity, allowing it to be used as a bait protein to screen the genes interacting with host.

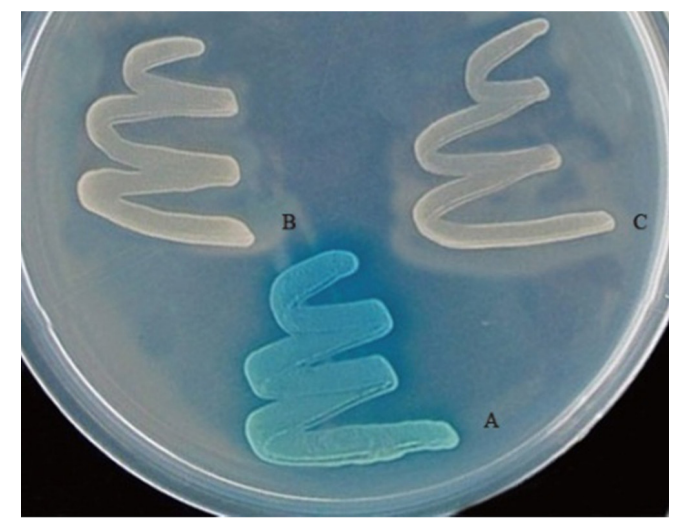

Figure 1. Growth of pGBK-OsPR $1 b p G B K-O s P R 1 b$ on an $S D /-T r p / X-\alpha-G a l$ plate. A. Positive control (bait protein with self-activation activity); B. pGBK-OsPR1b yeast strain; C. negative control (bait protein without selfactivation activity).

Based on the above detection results, the rice cDNA library was screened using the matched pair method with OsPR1b as the bait protein. A total of 182 clones were screened from the SD/Ade/-His/-Leu/-Trp plate and transferred onto a SD/-Ade/-His/-Leu/-Trp/X- $\alpha$-Gal plate. From the 96 blue clones that grew well, yeast plasmids were extracted and transformed into Escherichia coli DH5 $\alpha$. As indicated by sequencing, four factors may interact with OsPR1b (Figure 2A), i.e. a conserved hypothetical protein (Os01g0184200), similar to photosystem II $10 \mathrm{kDa}$ polypeptide (Os08g0200300), catalase (Os06g0306300), and resistance protein (Os01g0669100).

A
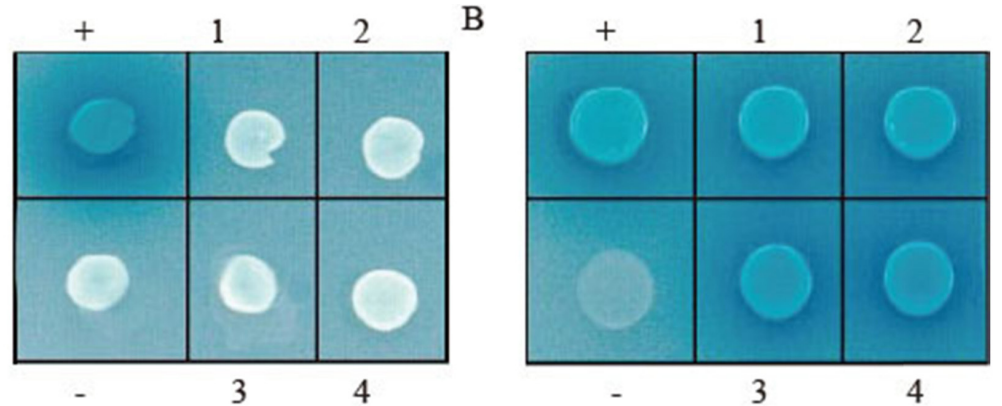

Figure 2. Yeast two-hybrid screening for self-activation activity (A) and interaction validation (B) of pGBKOsPR1b's interacting factors. (+) positive control; (-) negative control. 1-4: Conserved hypothetical protein (Os01g0184200), similar to photosystem II 10-kDa polypeptide (Os08g0200300), catalase (Os06g0306300), and resistance protein (Os01g0669100). 
The host factors obtained were transformed into the GOLD yeast strain to analyze their self-activation activities on a $\mathrm{SD} / \mathrm{-Leu} / \mathrm{X}-\alpha-\mathrm{Gal}$ plate. Since they were also colorless, as was the negative control (Figure 2B), they had no such activities. Subsequently, bait vector plasmid pGBK-OsPR $1 b$ and the factors were co-transformed into the GOLD yeast strain and cultured on an $\mathrm{SD} /-\mathrm{Ade} /-\mathrm{His} / \mathrm{-Leu} / \mathrm{-Trp} / \mathrm{X}-\alpha-$ Gal plate for 3-5 days. The host factors interacted with OsPR $1 b$, as evidenced by the colonies' bright blue colors resembling that of the positive control.

\section{Preparation of OsPR1b homozygous plants}

Overexpression of genes has commonly been used to study their functions. $\mathrm{T}_{0}$ generation plants were obtained by $A$. tumefaciens-mediated genetic transformation, transformant screening, and the regeneration of transgenic plants, which yielded plants with the separation ratio of 3:1 after PCR identification and screening. The $\mathrm{T}_{3}$ generation homozygous plants were subjected to quantitative fluorescent PCR detection, of which Line 7, which overexpressed OsPR1b 20 times was selected for subsequent experiments (Figure 3).

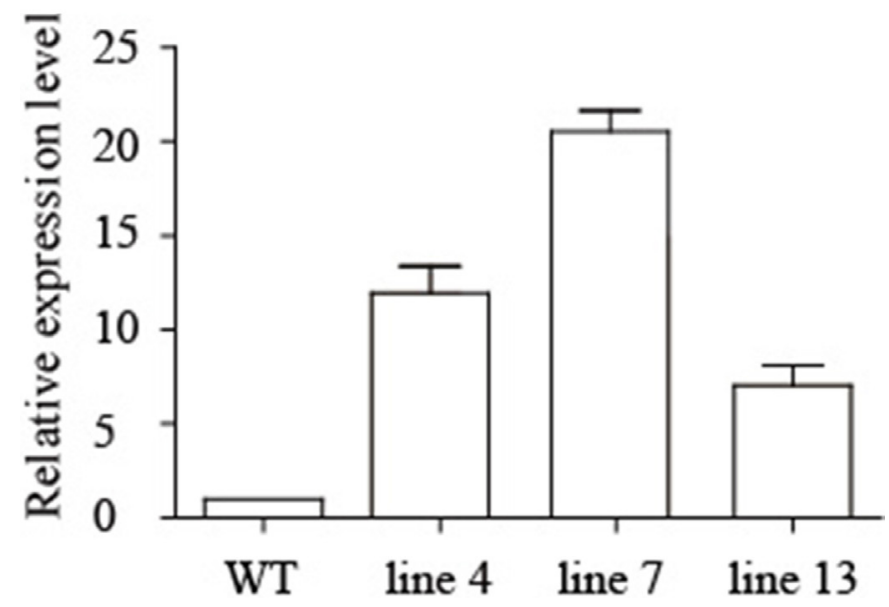

Figure 3. Semi-quantitative PCR detection of $\mathrm{T}_{3}$ generation lines overexpressing OsPR1b.

\section{Inducible expression characteristics of OsPR1b}

We then inoculated the leaves of two-week-old homozygous seedlings with Xoo P8 and treated their roots with SA, JA, and ET, aiming to analyze the interactions between rice pathogenic bacteria and the OsPR1b gene, as well as the roles of the three defense-related signaling molecules $(0.1 \mathrm{mM}$ each). After $12 \mathrm{~h}$ of treatment, the leaves were subjected to quantitative fluorescent PCR to detect the expression changes of OsPR1b (Figure 4). Compared with the control group that was inoculated with water, inoculation with a mixture of water and pathogenic bacteria hardly affected the expression level of OsPR1b gene, while cotreatment with SA and pathogenic bacteria slightly upregulated the expression level. However, cotreatment with JA or ET and pathogenic bacteria managed to significantly upregulate the expression level of the OsPR1b gene by 4.8 or 5.7 fold. 


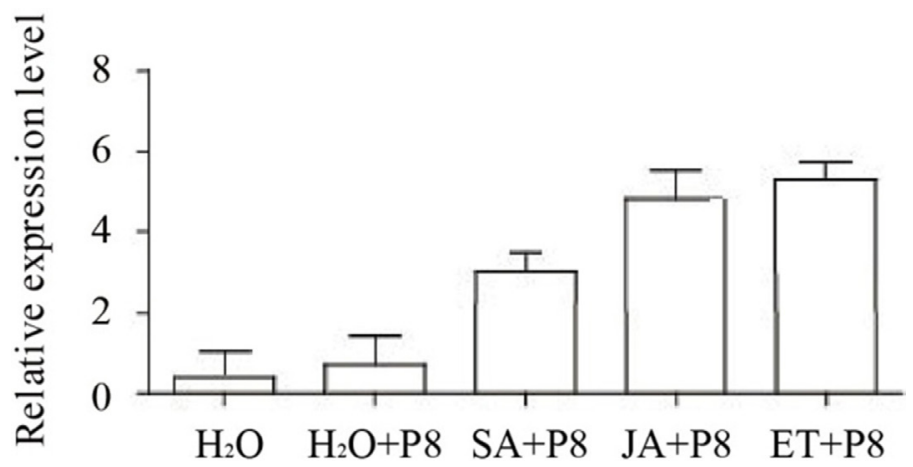

Figure 4. Expression levels of OsPR $1 \mathrm{~b}$ gene after $12 \mathrm{~h}$ of treatments with Xoo P8, SA, JA, and ET.

\section{DISCUSSION}

As an important food crop, rice activates, upon invasion with pathogenic bacteria or stimulation with physical injury, the expression of downstream genes by inducing self-defense responses. OsPR $1 \mathrm{~b}$ is a marker gene, the expression of which is silenced in most healthy plant leaves (Agrawal et al., 2001). With the well-established yeast two-hybrid assay method (Zhou et al., 2007), four host interacting factors were screened from the rice cDNA library by using OsPR $1 b$ as the bait protein, of which catalase $(\mathrm{Os} 06 \mathrm{~g} 0306300)$ catalyzes the decomposition of hydrogen peroxide into oxygen and water, and participates in many disease-resistance and stress-tolerance signaling pathways in plants (Willekens et al., 1997; Yang et al., 1997; Kruger et al., 2002). Moreover, resistance protein (Os01g0669100) further verified that OsPR1b may be associated with the disease resistance pathways of plants.

Elevating the level of endogenous SA barely affected the defense response of rice (Yuan et al., 2007), and the expression of PR genes in SA-deficient NahG rice was not remarkably decreased (Yang et al., 2004). Similarly, in this study, the expression level of OsPR1b was not significantly augmented by exogenously applying SA $12 \mathrm{~h}$ after invasion by pathogenic bacteria. Nevertheless, treating rice seedlings with JA induced more PR genes to be expressed (Browse, 2009). After $12 \mathrm{~h}$ of invasion by pathogenic bacteria, exogenous application of either JA or ET (Broekaert et al., 2006; Helliwell et al., 2013) successfully enhanced the resistance of rice to bacterial blight. It is likely that JA and ET managed to do so by dramatically increasing the expression level of the OsPR1b gene.

PR genes, which are sensitive, are affected by many unknown factors during expression, and the detailed regulatory mechanisms in rice still require in-depth study.

\section{ACKNOWLEDGMENTS}

We thank all the coauthors for their contribution.

\section{REFERENCES}

Agrawal GK, Rakwal R, Jwa NS and Agrawal VP (2001). Signaling molecules and blast pathogen attack activates rice OsPRIa and OsPR $1 b$ genes: A model illustrating components participating during defence/stress response. Plant Physiol. Biochem. 39: 1095-1103. 
Broekaert WF, Delaure SL, De Bolle MF and Cammue BP (2006). The role of ethylene in host-pathogen interactions. Annu. Rev. Phytopathol. 44: 393-416.

Browse J (2009). Jasmonate passes muster: a receptor and targets for the defense hormone. Annu. Rev. Plant Biol. 60: 183-205.

Gnanamanickam SS, Priyadarisini VB, Narayanan NN, Vasudevan P, et al. (1999). An overview of bacterial blight disease of rice and strategies of its management. Curr. Sci. India 77: 1435-1444.

Helliwell EE, Wang Q and Yang Y (2013). Transgenic rice with inducible ethylene production exhibits broad-spectrum disease resistance to the fungal pathogens Magnaporthe oryzae and Rhizoctonia solani. Plant Biotechnol. J. 11: 33-42.

Kruger J, Thomas CM, Golstein C, Dixon MS, et al. (2002). A tomato cysteine protease required for Cf-2-dependent disease resistance and suppression of autonecrosis. Science 296: 744-747.

Nahar K, Kyndt T, De VD, Hofte M, et al. (2011). The jasmonate pathway is a key player in systemically induced defense against root knot nematodes in rice. Plant Physiol. 157: 305-316.

Willekens H, Chamnongpol S, Davey M, Schraudner M, et al. (1997). Catalase is a sink for $\mathrm{H} 2 \mathrm{O} 2$ and is indispensable for stress defence in C3 plants. EMBO J. 16: 4806-4816.

Yang Q, Reinhard K, Schiltz E and Matern U (1997). Characterization and heterologous expression of hydroxycinnamoyl/ benzoyl-CoA:anthranilate N-hydroxycinnamoyl/benzoyltransferase from elicited cell cultures of carnation, Dianthus caryophyllus L. Plant Mol. Biol. 35: 777-789.

Yang Y, Qi M and Mei C (2004). Endogenous salicylic acid protects rice plants from oxidative damage caused by aging as well as biotic and abiotic stress. Plant J. 40: 909-919.

Yuan Y, Zhong S, Li Q, Zhu Z, et al. (2007). Functional analysis of rice NPR1-like genes reveals that OsNPR1/NH1 is the rice orthologue conferring disease resistance with enhanced herbivore susceptibility. Plant Biotechnol. J. 5: 313-324.

Zhou F, Wu G, Deng W, Pu Y, et al. (2007). Interaction of rice dwarf virus outer capsid P8 protein with rice glycolate oxidase mediates relocalization of P8. FEBS Lett. 581: 34-40. 